\title{
Farmaco equivalente, sicurezza del paziente e profili di responsabilità
}

\author{
- Luciana Pazzagli ${ }^{1}$, Anna Maria Cavallo ${ }^{2}$, Federico Romagnoli ${ }^{3}$, Nadia Palazzi $^{3}$, Giovanni Ceriello ${ }^{3}$, \\ Marianna Pulice ${ }^{4}$
}

Centro di Farmacovigilanza, Azienda Sanitaria Firenze

Medicina Generale, Azienda Sanitaria di Firenze

Farmacia di Continuità, Azienda Sanitaria di Firenze

Avvocato, Foro di Cosenza

\begin{abstract}
A diabetic patient under oral therapy with a generic drug had a sudden indisposition and hyperglycaemia due to the consumption of a tablet of another equivalent drug product - same active principle - with evident lack of therapeutic effect. The patient herself solved the problem by interrupting the new generic drugs and taking the one normally used. The general practitioner signalled the event to the Pharmacovigilance Centre that, in turns, signalled it to the Agenzia Italiana del Farmaco (AIFA), appointed to execute the controls. The case described is paradigmatic to discuss the responsibilities of the different actors involved (general practitioner, pharmacist, AIFA, and pharmaceutical industry).
\end{abstract}

Keywords: equivalent drug product, ineffectiveness, adverse event, responsibility

Equivalent drug product, patient's safety and profiles of responsibility

Pratica Medica \& Aspetti Legali 2010; 4(3): 119-127

\section{INTRODUZIONE}

Descriviamo in questo articolo un caso reale, osservato presso l'Azienda Sanitaria di Firenze, di mancato effetto terapeutico da farmaco equivalente. Il caso si presta a riflessioni più generali sulle criticità relative a questa categoria di farmaci e alla possibilità che l'inefficacia possa portare a un ipotetico evento grave e/o fatale per il soggetto interessato. Al tempo stesso il rischio correlato rimanda a eventuali responsabilità, proiettando il commento in ambito legale. Il tutto nella consapevolezza che la diffusione delle informazioni aumenti le conoscenze nei professionisti sanitari e che ciò apporti un beneficio alla sicurezza dei pazienti.

Infatti, l'immissione in commercio di un numero sempre maggiore di prodotti equivalenti se da una parte alletta i policy makers per i risparmi che generano rispetto ai farmaci branded, dall'altra richiede adeguate conoscenze per l'uso.

\section{DESCRIZIONE DEL CASO}

Una signora di 74 anni affetta da diabete mellito di tipo 2, in terapia da tempo con un farmaco ipoglicemizzante orale generico, si reca in farmacia con la specifica prescrizione medica.

A causa della mancanza in farmacia del prodotto prescritto, la signora ritira un generico equivalente diverso da quello abitualmente utilizzato (prodotto da un'altra azienda farmaceutica) per sostituzione da parte del farmacista all'atto della dispensazione, come previsto per legge in questa evenienza, su consenso del cittadino.

Dopo l'assunzione di una compressa, la paziente avverte una sensazione di malessere con sintomi simili a quelli provocati da un'elevata glicemia.

Effettuate diverse misurazioni sul sangue capillare con strisce diagnostiche, e avendo riscontrato valori elevati non tendenti a diminuire in funzione dell'assunzione del farmaco. 
Disponendo di alcune compresse del farmaco equivalente precedentemente utilizzato, la paziente ne assume una e i valori glicemici rientrano nella norma.

L'andamento "acuto" di questo evento, il rechallenge e il dechallenge individuano il nesso di causalità nel farmaco equivalente sostituito e confermano la non interscambiabilità tra equivalenti.

\section{ESITI}

L'esperienza nella gestione della malattia e la prontezza della signora hanno sventato ogni possibile esito clinicamente grave e/o fatale.

La preoccupazione del medico curante si è tradotta quindi in una scheda di sospetta reazione avversa al farmaco, per mancanza di efficacia, inoltrata al Centro di farmacovigilanza dell'Azienda Sanitaria di Firenze.

Ciò ha permesso all'autorità regolatoria (AIFA, Agenzia Italiana del Farmaco) di avviare il procedimento previsto per chiarire il caso e verificare le caratteristiche del farmaco, attraverso l'Istituto Superiore di Sanità. Il procedimento è attualmente ancora in corso.

È comunque evidente che, se la paziente non avesse provveduto a gestire e risolvere prontamente la mancanza di effetto terapeutico, sarebbe potuta incorrere in un evento avverso grave che avrebbe potuto metterla in pericolo di vita. Nell'ipotesi più remota che l'esito fosse fatale, considerando criticità e problemi aperti sui farmaci generici/equivalenti, affiorano immediate domande sulle possibili responsabilità: di che tipo e a carico di chi? (medico, farmacista, paziente, azienda farmaceutica produttrice o AIFA?). La seconda parte di questo articolo prende appunto in esame tali possibili responsabilità.

\section{UNO SGUARDO ALLA NORMATIVA FARMACEUTICA}

\section{GENERICI}

Sono detti generici i farmaci "simili" a prodotti originali con marchio brevettato dalle Aziende Farmaceutiche (branded) non più protetti dalla copertura brevettuale, con un prezzo inferiore di almeno il $20 \%$ rispetto alla specialità.

Con la Legge n. 405 del 16 novembre 2001 il generico assume il ruolo di farmaco di riferimento per il sistema di rimborso dei medicinali non coperti da brevetto, aventi identiche composizioni in principi attivi, forma farmaceutica, via di somministrazione, modalità di rilascio e infine dosi unitarie.
Da allora la normativa italiana consente e incentiva l'interscambiabilità tra un prodotto di marchio e l'equivalente generico, permettendo al farmacista di dispensare un generico al posto del medicinale branded prescritto dal medico, se questi non indica esplicitamente il suo diniego in ricetta o se lo stesso diniego proviene dal cittadino.

\section{EQUIVALENTI}

Con la Legge n. 149 del 26 luglio 2005 viene introdotta in Italia la definizione di "equivalente" allo scopo di precisare la definizione di equivalenza terapeutica del farmaco generico.

Lart. 1 bis della citata legge dispone: «I medicinali con obbligo di prescrizione medica di cui all'art. 7, comma 1 , del DL 18/9/2001, n. 347, convertito con modificazioni, dalla legge 16/11/2001, n. 405, ad esclusione di quelli che hanno goduto di copertura brevettuale, sono definiti "medicinali equivalenti"».

\section{BIOEQUIVALENTI}

Sono bioequivalenti i medicinali che hanno equivalenza farmaceutica, stessa biodisponibilità e differenze non rilevanti in termini di efficacia e sicurezza del farmaco originator, ovvero che rispondono ai seguenti requisiti:

- contengono la stessa quantità di principio attivo;

- hanno la stessa forma farmaceutica (anche con eccipienti diversi);

- posseggono standard di qualità identici o comparabili;

- sono formulati per la stessa via di somministrazione.

L'equivalenza terapeutica non è valutata su parametri clinici di efficacia, ma sulla biodisponibilità sistemica di due prodotti.

I test di bioequivalenza si basano sul confronto statistico di parametri farmacocinetici che caratterizzano la biodisponibilità dei due prodotti.

Generalmente vengono usati i parametri AUC, $\mathrm{C}_{\text {max }}$ e $t_{\max }$ (area sotto la curva concentrazione/tempo, massima concentrazione plasmatica e tempo per raggiungere la massima concentrazione), ma quando ciò non è fattibile si può ricorrere a parametri relativi all'escrezione urinaria o a parametri farmacodinamici direttamente correlabili con l'esposizione al farmaco.

Le differenze di biodisponibilità tra due prodotti essenzialmente simili non devono superare il range di variabilità ritenuto compatibile con l'equivalenza terapeutica.

Secondo le linee guida dell'Agenzia Europea dei Medicinali (EMA) due farmaci sono equivalenti se sia l'intervallo di confidenza al $90 \%$ relativo al rap- 
porto tra le rispettive AUC, sia l'intervallo di confidenza al $90 \%$ relativo al rapporto tra le rispettive $\mathrm{C}_{\max }$ cadono nel range $0,80-1,25$.

La documentazione che attesta la bioequivalenza di un farmaco generico rispetto a una specialità medicinale ai fini dell'immissione in commercio (AIC) non è richiesta quando:

- la domanda di AIC sia presentata dal titolare della specialità a brevetto scaduto o da un suo licenziatario;

- i metodi di fabbricazione e di officina di produzione siano identici;

- la via di somministrazione e le caratteristiche rendano le variazioni di composizione quali-quantitativamente irrilevanti rispetto alla biodisponibilità (es. fiale endovena);

- l'AIC è concessa dall'AIFA seguendo la procedura di registrazione nazionale (DL 219/2006).

\section{BIOSIMILARI}

Sono prodotti farmacologicamente analoghi ai farmaci realizzati con tecniche biotecnologiche che hanno oltrepassato il periodo di copertura brevettuale.

Non potendo essere bioequivalenti, questi farmaci necessitano, per l'autorizzazione all'immissione in commercio, di presentare anche gli studi clinici in quanto sono prodotti con l'uso di linee cellulari differenti da quelle del prodotto originator.

Il prezzo non può essere per questi farmaci troppo inferiore a quello dell'originator, essendo la produzione biotecnologica di sostanze proteiche molto costosa.

\section{CRITICITÀ LEGATE AI FARMACI EQUIVALENTI}

Per essere perfettamente identiche due specialità medicinali, oltre ad avere gli stessi principi attivi, devono avere i medesimi eccipienti ed essere state sottoposte alla stessa lavorazione tecnologica (avere, ad esempio, la stessa granulometria). Ciò significa, sostanzialmente, che per essere identici due farmaci devono essere licenziati dallo stesso impianto di produzione. Per questo motivo il farmaco equivalente non viene definito identico al prodotto branded, ma "essenzialmente simile".

Tali minime differenze non devono comportare risultati terapeutici significativamente differenti nella popolazione. Tuttavia proprio la presenza di queste potenziali variazioni porta alcuni Autori a proporre di evitare di sostituire un prodotto con un altro, anche se similare. Nel caso degli antiepilettici, ad esempio, Perrucca e colleghi sconsigliano di cambiare generico nei pazienti che hanno ottenuto una remissione dei sintomi, sostenendo: "In patients treated with a generic, it is preferable to avoid its substitution with products (including other generics) from different manufacturers» [1]. Analogamente, in ambito cardiovascolare, molti editoriali si sono dimostrati contrari alla sostituibilità $(6$ su 14 di quelli pubblicati nel biennio 2006-2008, pari al $43 \%$ ), e ciò indipendentemente dal fatto che non vi siano evidenze chiare sulla rilevanza clinica delle differenze tra generico e prodotto branded [2].

Le differenze di formulazione tra la specialità medicinale originator e l'equivalente possono creare problemi di vario tipo:

- diverso rilascio del principio attivo in relazione alla forma farmaceutica;

- problemi di allergia o intolleranza ai diversi tipi di sostanze veicolanti, eccipienti o impurità;

- diversa solubilizzazione o formazione di precipitati per ricostituzione di forme iniettabili;

- variazioni di efficacia per farmaci con ristretto margine terapeutico (es. anticoagulanti orali, antiepilettici, antiaritmici, digitalici; per questi anche piccole variazioni di biodisponibilità possono comportare sensibili variazioni);

- mancanza o ridotto effetto terapeutico per switch da un originator a un equivalente o da un equivalente a un altro;

- possibile esposizione a maggior rischio di reazioni avverse.

Da non sottovalutare poi l'utilizzo di materie prime di scarsa qualità (specie per biosimilari) e i diversi processi di produzione.

In relazione alla bioequivalenza vi sono problemi sottostimati o trascurati:

- i test eseguiti consentono di stimare una "bioequivalenza media di popolazione" e non una "bioequivalenza individuale";

- i test sono fatti tra il singolo generico e il prodotto branded: ciò non garantisce che più generici di uno stesso originator siano tra loro bioequivalenti;

- gli intervalli di accettazione della bioequivalenza sono "accettabili"?

Al di là delle differenze oggettive tra generico e prodotto branded, alcuni studi evidenziano la necessità di tenere in considerazione anche la percezione dei prodotti farmaceutici da parte dei pazienti. Il medico e il farmacista hanno il compito di illustrare in modo chiaro il significato di equivalenza e le caratteristiche del prodotto generico. Tuttavia sono anche tenuti a prender in considerazione il fatto che talvolta le credenze del paziente in relazione a questi farmaci possano influire sul 


\begin{tabular}{|ll|}
\hline \multicolumn{1}{|c|}{ Provredimento } & \multicolumn{1}{c|}{ Note } \\
$\begin{array}{l}\text { Parere del Consiglio di } \\
\text { Stato n. 3992/06 del } \\
20 \text { giugno } 2007\end{array}$ & $\begin{array}{l}\text { In merito a un quesito della Regione } \\
\text { vale per i farmaci biotecnologici. Per il } \\
\text { suo pronunciamento il Consiglio di Stato } \\
\text { si è avvalso del parere del Ministero della } \\
\text { Salute per cui il concetto di medicinale } \\
\text { generico e per definizione equivalente } \\
\text { non può essere applicato ai farmaci } \\
\text { biologici e biotecnologici }\end{array}$ \\
\hline $\begin{array}{l}\text { Sentenza TAR Emilia } \\
\text { Romagna 25/06/2007, } \\
\text { n. 1235 e sentenza } \\
\text { TAR Emilia Romagna } \\
\text { sez. II, 2/01/2009 }\end{array}$ & $\begin{array}{l}\text { Si limitano a negare che l'equivalenza } \\
\text { farmaci biologici. L'efficacia dovrà essere } \\
\text { accertata di volta in volta in relazione a } \\
\text { una o più indicazioni terapeutiche sulla } \\
\text { base delle relative evidenze cliniche }\end{array}$ \\
\hline $\begin{array}{l}\text { Decisione del } \\
\text { Consiglio di Stato n. } \\
691 / 2010 \text { REG. DEC. }\end{array}$ & $\begin{array}{l}\text { Accoglimento ricorso contro il Centro } \\
\text { Servizi Condivisi Regione Friuli Venezia } \\
\text { Giulia, per lotto in cui si mettono a } \\
\text { gara ueritropoietina alfa 40.000 Ul ed } \\
\text { eritropoietina beta 30.000» }\end{array}$ \\
\hline
\end{tabular}

Tabella I. Alcuni dei pareri espressi ad oggi a livello italiano

suo accordo a sostituire il prodotto. Uno studio in tal senso è stato condotto da Figueiras e colleghi, e ha concluso che la disponibilità dei pazienti ad accettare la sostituzione con il generico può essere influenzata sia dalla patologia diagnosticata (influenza, asma o angina pectoris) sia dai fattori socio-demografici [3].

\section{PROFILI DI RESPONSABILITÀ}

La valutazione giuridica delle forme di responsabilità medica richiede l'analisi puntuale di istituti fondamentali del diritto, oggetto di contrasti giurisprudenziali e dottrinali da diverso tempo. Lattribuzione in concreto di una forma di responsabilità ai soggetti coinvolti in un caso di malpractice medica 0 , più ampiamente, sanitaria è piuttosto complessa e, comunque, necessita di una valutazione in concreto e non di certo in via astratta. Nel caso sopra descritto i soggetti coinvolti sembrerebbero il farmacista, il medico, l'AIFA e la casa farmaceutica, a parte, naturalmente, il danneggiato, ovvero la paziente affetta da diabete mellito di tipo 2 .

\section{IL FARMACISTA}

Il farmacista esercita una professione intellettuale sanitaria che, nel tempo, è andata cambiando in maniera radicale.

In origine l'attività si concretizzava in prevalenza nell'approntamento, su ricetta medica, dei c.d. prodotti galenici, preparati medicamentosi prodotti direttamente in farmacia. L'alta professionalità necessaria per tutto questo è presto venuta meno con la diffusione delle specialità medicinali preconfezionate, c.d. industriali, così trasformando la figura del farmacista sempre più in un imprenditore commerciale.

È a seguito di tale trasformazione che si legge nelle sentenze che «l'attività di vendita al dettaglio al pubblico di farmaci (indipendentemente dal fatto che il costo sia o meno a carico del Servizio sanitario pubblico) costituisce un'attività economica commerciale di carattere imprenditoriale. Ad essa devono pertanto applicarsi il principio di libertà di iniziativa economica privata sancito dall'art.41 Cost. e il principio di libera concorrenza sancito dal Trattato 25 marzo 1957, restando la tutela del consumatore assorbita, alla stregua di un criterio di proporzionalità e di ragionevolezza, dalla presenza al banco di un farmacista» [4].

Ma, come sottolineato da autorevole dottrina (A. Falzea), i farmaci vanno a incidere sul bene essenziale della salute ed è per questo che il legislatore ha comunque riservato il servizio farmaceutico solo a chi ha delle specifiche cognizioni professionali della materia ovvero "una conoscenza altamente specializzata, un addestramento ben controllato nonché l'impegno di una intensa attenzione».

Nel testo Unico delle leggi sanitarie [5], all'art. 122 , è prevista, infatti, la vendita dei medicinali a dose o forma di medicamento solo da parte dei farmacisti ed esclusivamente nella farmacia, sotto la responsabilità del titolare della medesima.

Dunque, il farmacista è sì un imprenditore ma è, anche e principalmente, un esercente una professione sanitaria rispetto alla quale deve osservare la massima diligenza; è un professionista che assume un'obbligazione di facere, l'obbligo di un'attività complessa e articolata e non una semplice obbligazione di dare (un prodotto, il farmaco, dietro corrispettivo).

Ma quando il farmacista ha davanti a sé una ricetta medica (obbligatoria ex art. 122 del Testo unico delle leggi sanitarie e strumento di controllo e di garanzia tra il prescrivente e il dispensatore) il dovere di diligenza si può atteggiare in maniera diversa: se si tratta di sostanze velenose a dosi pericolose, l'art. 40 del Regolamento del Servizio Sanitario Farmaceutico, n. 1706/1938, prevede che il farmacista si possa rifiutare di somministrarle se il medico non ha dichiarato per iscritto che l'erogazione è sotto la sua responsabilità; se la sostanza non rientra in tale categoria non si può rifiutare, anche perché non può essere a conoscenza di tutta la situazione clinica della paziente e, dunque, delle esigenze terapeutiche precipue del caso; se il farmaco prescritto, come nel caso de quo, manca nella farmacia il farmacista può, con il consenso del paziente, cambiare il prodotto con un altro equivalente. 
Il prodotto "generico" (introdotto dall'art. 130, comma 3, della L. 28 dicembre 1995, n. 549) è una specialità medicinale "essenzialmente simile" a un prodotto il cui brevetto è scaduto e del quale imita la formulazione: ha, quindi, la stessa composizione quali-quantitativa di principio/i attivo/i e una forma farmaceutica equivalente.

Il termine "generico", già al'indomani della sua introduzione, si è dimostrato infelice, in quanto percepito dal pubblico come una sorta di rimedio passepartout ovvero un prodotto simile, ma non uguale, a uno o più medicinali indicati per la stessa patologia. Per questa ragione i prodotti "generici" sono stati ridefiniti, con la L. 149 del 26 luglio 2005, "equivalenti”. Infine, nel codice comunitario concernente i medicinali per uso umano (D. Lgs. 24 aprile 2006, n. 219), le "specialità medicinali", per evitare l'attribuzione di caratteristiche particolari al termine "specialità", sono state ridefinite "medicinali".

I farmaci equivalenti sono registrati e commercializzati con la denominazione comune internazionale del principio attivo seguito dal nome del produttore (titolare dell'autorizzazione all'immissione in commercio) e devono avere un prezzo inferiore di almeno il $20 \%$ rispetto a quello del prodotto innovatore o brand (con nome di fantasia). Dunque, la sostituzione del farmaco prescritto con uno equivalente è normalmente prevista e non può sorgere una forma di responsabilità per il farmacista qualora si dovesse riscontrare che il principio attivo dei due farmaci è lo stesso, come non sembra potersi mettere in dubbio nel caso in esame.

Addirittura il farmacista «non è tenuto a controllare l'indicazione della posologia del farmaco prescritto dal medico di base e, di conseguenza, non è responsabile dei danni subiti dal paziente a causa di un sovradosaggio nell'assunzione del medicinale» [6].

Alla luce di quanto detto, nessuna forma di responsabilità può ascriversi al farmacista che, come previsto dalla legge, ha cambiato il farmaco con un medicinale equivalente.

\section{IL MEDICO}

Per quel che riguarda il medico, il caso de quo non comporta forme di responsabilità anche perché, dai dati fattuali in nostro possesso, emerge che gli unici doveri a suo carico in tali fattispecie, che si concretizzano in un obbligo di segnalazione, sono stati assolti dal professionista.

La farmacovigilanza è, in effetti, un sistema di monitoraggio permanente nata dalla necessità di allargare il controllo dell'efficacia dei farmaci nel momento del loro uso su larga scala; l'obiettivo, pertanto, è quello di raccogliere informazioni sui farmaci, sulla loro efficacia e, in particolare, sul- le eventuali reazioni avverse. Fa capo all'Agenzia Italiana del Farmaco, un organismo di diritto pubblico che opera sulla base degli indirizzi e della vigilanza del Ministero della Salute, in autonomia, trasparenza ed economicità, con una funzione prettamente culturale, di conoscenza e che serve, altresì, come consulente tecnico-scientifico.

Il medico curante in questo caso ha segnalato la reazione avversa della paziente al centro di farmacovigilanza e questo all'AIFA che, in seguito, ha riscontrato oggettivamente dei problemi per il farmaco.

Il D.M. 27 febbraio 2001, recante le "Disposizioni da applicare in caso di rinvenimento di medicinali con difetti o contenenti corpi estranei”, prevede le modalità da seguire per le segnalazioni da parte di ospedali, personale sanitario, aziende produttrici o titolari di autorizzazioni all'immissione in commercio (AIC) e richiama i provvedimenti urgenti previsti in tali ipotesi quali il ritiro, il divieto di utilizzo, il campionamento, il sequestro cautelativo di uno o più lotti di prodotti medicinali difettosi. Dunque, anche quest'altro protagonista della vicenda può essere escluso da una qualsiasi forma di responsabilità.

\section{L'ISTITUTO SUPERIORE DI SANITÀ}

La L. n. 519/1973 ha attribuito all'Istituto Superiore di Sanità compiti attivi a tutela della salute pubblica e con la L. n. 833/1978, istitutiva del Servizio Sanitario Nazionale, è stato confermato per il Ministero della Sanità un ruolo primario nella programmazione del piano sanitario nazionale con compiti di indirizzo e coordinamento delle attività amministrative regionali delegate in materia sanitaria, nonché importanti funzioni in materia di produzione, sperimentazione e commercio dei prodotti farmaceutici e degli emoderivati (art. 6, lett. b e c).

Il D. L. n. 443/1987 è quello che stabilisce espressamente la sottoposizione dei medicinali alla c.d. "farmacovigilanza" da parte del Minisero della Sanità, che deve prevedere le modalità di esecuzione del monitoraggio sui farmaci a rischio ed emettere provvedimenti cautelari sui prodotti in commercio, e che, pertanto, ha un vero e proprio obbligo di controllo, direttiva e vigilanza anche strumentale alla funzione di programmazione e coordinamento in materia sanitaria.

L'omissione da parte del Ministero di attività funzionali alla realizzazione dello scopo per il quale l'ordinamento attribuisce il potere (concernente la salute pubblica) lo sottopone, quindi, a una forma di responsabilità extracontrattuale, quando dalla violazione del vincolo interno costituito dal dovere di vigilanza nell'interesse pubblico, il quale è strumentale e accessorio a quel potere, siano derivate violazioni dei diritti soggettivi dei terzi [7]. 
Alla luce di ciò, in molti giudizi, specie di risarcimento danni conseguenti a infezioni contratte da soggetti emotrasfusi per l'omessa vigilanza esercitata dall'Amministrazione sulla sostanza ematica negli interventi trasfusionali e sugli emoderivati, la responsabilità del Ministero della Salute viene inquadrata nella violazione della clausola generale di cui all'art. 2043 c.c. [8] e il Ministero viene chiamato in causa come soggetto legittimato passivo rispetto all'azione di responsabilità extracontrattuale spiegata.

Il passo immediatamente successivo alla chiamata in causa è la dimostrazione, però, del nesso causale tra la predetta violazione e il danno subìto.

Nel caso degli emoderivati il percorso è facilitato perché la verifica della sussistenza può condursi sulla base dei principi di diritto emanati dalle Sezioni Unite della Suprema Corte negli anni recenti su casi di infezioni epatiche che si assumevano essere state contratte proprio in conseguenza dell'assunzione di farmaci emoderivati [9]; nel caso, invece, di farmaci, e peraltro equivalenti, il D. L. 323/96 stabilisce che l'immissione in commercio viene autorizzata dal Ministero della Sanità sulla base della semplice presentazione di una documentazione che attesti la bioequivalenza rispetto a una specialità medicinale già autorizzata, con la stessa composizione quali-quantitativa dei principi attivi, la stessa forma farmaceutica e le stesse indicazioni terapeutiche. Ecco che allora la chiamata in causa e la dimostrazione del nesso causale diventano giuridicamente difficili!

\section{LA SOCIETÀ FARMACEUTICA}

Una volta valutate ed escluse, con una certa semplicità logica, prima ancora che giuridica, alcune posizioni, occorre adesso soffermarsi, con molta più attenzione e con maggiori momenti riflessivi, sulla responsabilità della società farmaceutica, alla quale però, pur assumendo la qualifica di produttore, non è dato applicare, secondo i principi giurisprudenziali attualmente vigenti, il Codice del Consumo.

In dottrina molto si discute, e ancora molto si discuterà, sulla responsabilità delle case farmaceutiche nell'eventualità di danni cagionati dall'assunzione di farmaci difettosi.

Le ricostruzioni teoriche prospettate in dottrina sono essenzialmente due: una responsabilità derivante dall'esercizio di attività pericolose ex art. 2050 c.c. e una responsabilità del produttore prevista e disciplinata dal c.d. Codice del Consumo. Come ogni scelta di impostazione giuridica le conseguenze non sono puramente terminologiche ma comportano rilevantissimi effetti sul piano pratico. In effetti, è la sussistenza stessa della responsabilità che cambia nell'uno e nell'altro caso: se si applica il D. Lgs. 206/2005 (Codice del Consu- mo), ex art. 118, la casa farmaceutica che immette sul mercato un farmaco, che allo stato delle conoscenze tecnico-scientifiche non risulta produttivo di danni, non è responsabile di eventuali pregiudizi successivi che non potevano essere scoperti prima; mentre, se si applica l'art. 2050 c.c., l'azienda sarà tenuta al risarcimento anche se il difetto non era conoscibile al momento dell'immissione, salvo che non provi di avere adottato tutte le misure idonee a evitare il danno.

Ma vediamo più nel particolare.

La disciplina speciale della responsabilità civile del produttore per danni causati da difetti del prodotto ha avuto origine nel nostro Paese dalla Direttiva comunitaria n. 374/1985 (integrata dalla Direttiva 34/1999, attuata con D. Lgs. 2 febbraio 2001, n. 25 , che ha esteso l'ambito della normativa ai prodotti agricoli), la quale è stata recepita con D.P.R. 374/1985.

Il nostro ordinamento non prevedeva la fattispecie della responsabilità del produttore perché il contesto economico proprio della codificazione del 1942 era per lo più improntato sull'artigianato e sulla vendita diretta; solo con l'industrializzazione avanzata è scaturita una realtà quasi diametralmente opposta a quella regolamentata nel codice civile. Alla luce di ciò, la giurisprudenza ha cercato di soddisfare le esigenze dei consumatori emergenti dalla nuova realtà sociale mediante l'applicazione dell'apparato normativo esistente ovvero la responsabilità extracontrattuale ex art. 2043 c.c. e gli artt. 2049, 2050 e 2051 c.c., opportunamente interpretati in funzione di una logica di evidente favore nei confronti del consumatore.

Il caso giurisprudenziale che notoriamente ha segnato un punto di partenza è quello deciso con la sentenza del 25 maggio 1964, n. 1270, che ha visto contrapposti Schettini e la Saiwa in merito a un prodotto avariato, in cui la Cassazione ha, innanzitutto, escluso la responsabilità del venditore: altrimenti la vicenda si sarebbe risolta in un'assurda responsabilità oggettiva e che avrebbe, comunque, offerto ben scarsa protezione al consumatore sia per i limiti di decadenza e prescrizione di cui all'art. 1495 c.c. sia per la facile dimostrazione da parte del produttore di aver ignorato senza colpa i vizi della cosa; l'avaria è stata invece ricollegata, mediante un ragionamento logico presuntivo, alla difettosa fabbricazione del prodotto quale sua unica possibile causa, aggirando così l'ostacolo della responsabilità contrattuale non configurabile nei confronti del produttore, che si pone come terzo soggetto, affermando, invece, la sua responsabilità per illecito aquiliano cagionato agli acquirenti. Occorreva, però, sganciarsi dal criterio della responsabilità per colpa e, così, si è utilizzato il percorso delineato dall'art. 2049 c.c. ovvero l'applicazione non diretta (perché ciò postulerebbe la colpa del preposto o commesso) ma analogica: fonte del danno in tal modo è l'intera organizzazione dell'impresa intesa 
oggettivamente nella sua totalità. Inoltre, è stato ampliato il concetto di attività pericolosa e il principio dell'art. 2050 c.c. è stato utilizzato, altresì, in materia di somministrazione di emoderivati infetti e di danno da prodotti farmaceutici $[10,11]$.

La Direttiva comunitaria 374/1985 ha stabilito che la tutela della concorrenza e della libera circolazione delle merci a livello comunitario passa anche e principalmente attraverso l'affermazione della responsabilità del produttore e ha affermato una forma di responsabilità oggettiva (art 1 Dir. 374/1985 trasfusa nell'art. 1 D.P.R. 224/1988): unico presupposto dell'applicazione della responsabilità oggettiva extracontrattuale al fabbricante, in effetti, è la nozione di difettosità e il nesso di causalità tra prodotto e fatto dannoso, senza dare alcun rilievo agli elementi di tipo soggettivo.

Tale disciplina, attualmente vigente nel nostro Paese, è stata trasposta, nel 2005, nel c.d. Codice del Consumo (D. Lgs. 206/2005) che stabilisce che «il produttore è responsabile del danno cagionato da difetti del suo prodotto» [12] (art. 114) e, dopo aver definito il prodotto [12] (art. 115) e precisato i casi in cui del danno cagionato risponde il fornitore [12] (art. 116), prevede una vera e propria presunzione di responsabilità in capo al produttore stesso [12] (combinato disposto degli artt. 117 e 120, già artt. 5 e 8 del D.P.R. 224/88). Infatti si legge nell'art. 117 del Codice del Consumo: «Un prodotto è difettoso quando non offre la sicurezza che ci si può legittimamente attendere tenuto conto di tutte le circostanze, tra cui: a) il modo in cui il prodotto è stato messo in circolazione, la sua presentazione, le sue caratteristiche palesi, le istruzioni e le avvertenze fornite; b) l'uso al quale il prodotto può essere ragionevolmente destinato e i comportamenti che, in relazione ad esso, si possono ragionevolmente prevedere; c) il tempo in cui il prodotto è stato messo in circolazione. 2. Un prodotto non può essere considerato difettoso per il solo fatto che un prodotto più perfezionato sia stato in qualunque tempo messo in commercio. 3. Un prodotto è difettoso se non offre la sicurezza offerta normalmente dagli altri esemplari della medesima serie». Inoltre, l'art. 120 del Codice ripartisce l'onere probatorio stabilendo che «il danneggiato deve provare il difetto, il danno, e la connessione causale tra difetto e danno. 2. Il produttore deve provare i fatti che possono escludere la responsabilità secondo le disposizioni dell'articolo 118. Ai fini dell'esclusione da responsabilità prevista nell'articolo 118 , comma 1, lettera b), è sufficiente dimostrare che, tenuto conto delle circostanze, è probabile che il difetto non esistesse ancora nel momento in cui il prodotto è stato messo in circolazione. 3. Se è verosimile che il danno sia stato causato da un difetto del prodotto, il giudice può ordinare che le spese della consulenza tecnica siano anticipate dal produttore».
Ma il punto cruciale di questa normativa è nell'art. 118 del Codice del Consumo perché, nel recepire le cause di esclusione della responsabilità, tra le quali il c.d. "rischio di sviluppo", non ha escluso da tale ipotesi, come hanno fatto altri legislatori europei (ad es. il legislatore spagnolo nella legge 22/1994 o anche quello finlandese), i produttori farmaceutici.

Da qui sorgono essenzialmente le critiche contro le case farmaceutiche che cercano di qualificare l'attività di produzione farmaceutica come un'attività non pericolosa per poter equiparare la responsabilità del produttore di farmaci a quella di un comune produttore: sorgerebbero gravi conseguenze per il paziente danneggiato in quanto, stante l'esenzione dal rischio di sviluppo in capo al produttore, il consumatore non avrebbe il risarcimento se il difetto del prodotto non era riconoscibile e rilevabile al momento della sua immissione sul mercato.

È per questa ragione che nel nostro Paese si è cercato per l'ennesima volta di sopperire a un'attività legislativa lacunosa o, per essere più precisi, poco attenta alle fattispecie concrete da inserire nell'ambito applicativo di una nuova legge, per quanto solo di recepimento di una direttiva comunitaria.

E così si è finiti per cercare la migliore tutela del cittadino mediante interpretazioni laboriose e forse un po' forzate.

Innanzitutto, anche dopo l'entrata in vigore del D.P.R. 224/88 sulla responsabilità del produttore, i giudici di merito e la suprema corte hanno continuato ad applicare ai casi di risarcimento dei danni da "farmaco difettoso" la disposizione ex art. 2050 c.c. [13] precisando altresì che "non incorre nella presunzione di responsabilità ex art. 1 comma 4 D.P.R. n. 224 del 1988 chi non sia produttore del farmaco ma semplice distributore dello stesso, confezionato con indicazione precisa del produttore» [14] (nella specie, la società distributrice in Italia di farmaco prodotto da diversa società, con sede in Francia, è stata esentata dalla presunzione di responsabilità di cui al D.P.R. n. 224 del 1988 , e chiamata a rispondere del danno subito dall'utente del farmaco secondo la generale previsione dell'art. 2050 c.c.).

Il punto sul quale le case farmaceutiche si sono battute, per la sua affermazione nelle aule di tribunale, è la non qualificazione dell'attività di produzione $\mathrm{e}$ commercializzazione di un farmaco come pericolosa. Ma la giurisprudenza, sin dagli anni ' 80 , ha sostenuto la pericolosità dell'attività di produzione, innanzitutto, dei farmaci contenenti gammaglobuline umane sostenendo che «è da ritenere esercente un'attività pericolosa colui che produce una sostanza potenzialmente lesiva - nella specie emoderivata - da utilizzare per comporre un farmaco destinato ad essere iniettato nell'organismo umano; pertanto ai sensi dell'art. 2050 c.c. egli è responsa- 
bile in solido con il produttore finale dei danni derivati dall'uso del medicinale - e pur se questi abbia omesso di verificarne l'innocuità, perché tale causa prossima non ha efficienza causale autonoma per la produzione dell'evento - salvo che provi di aver impiegato ogni cura e misura secondo le conoscenze tecniche e scientifiche esistenti, atte ad impedirlo (Nella specie ricerca del virus con il metodo RIA)» [10,11,15-17]. Ma le Corti hanno inteso tale principio applicabile più in generale anche a tutti i farmaci non emoderivati si legge infatti: "L'importazione e la distribuzione di farmaci è attività che deve considerarsi pericolosa non solo perché tale è considerata dal legislatore, ma anche perché ad essa è connaturale una apprezzabile potenzialità lesiva, in quanto sostanzialmente diffonde nel pubblico un rilevante pericolo di malattia, derivato dalla natura del mezzo adoperato» [14].

Ecco che allora la posizione processuale delle aziende produttrici di farmaci diventa delicata perché le imprese farmaceutiche sono responsabili, ai sensi dell'art. 2050 c.c., dei danni derivanti da patologie prodotte dall'uso di un farmaco nell'ipotesi in cui, pur avendo ottemperato alle disposizioni normative vigenti, non dimostrino di avere adottato ogni cautela idonea a impedire l'evento. Infatti, poiché la presunzione di responsabilità prevista dal citato art. 2050 per le attività pericolose, tra le quali rientra anche quella di produzione di farmaci, può essere vinta solo dalla prova di avere adottato tutte le misure idonee a evitare il danno, non basta, per escludere la responsabilità dell'esercente l'attività pericolosa, la prova negativa di non avere commesso alcuna violazione di legge o di comune prudenza, ma occorre quella positiva di avere impiegato ogni cura o misura atta a impedire l'evento dannoso [18].

Le sezioni unite hanno affermato che «quanto al concetto di attività pericolosa per sua natura, è giurisprudenza costante di questa Corte che debba intendersi non solo quell'attività che espressamente è ritenuta tale dalla legge (segnatamente quella di P.S.), ma anche ogni altra attività, che pur non essendo legislativamente qualificata come tale, tuttavia abbia una pericolosità intrinseca $o$ relativa ai mezzi impiegati $[10,19]$. La qualifica di pericolosità di un'attività dipende, quindi, da una valutazione empirica: la quantità di pericolo che la connota. Un primo indice rilevatore della pericolosità si ha quando dall'esercizio dell'attività derivi un'elevata probabilità o una notevole potenzialità dannosa, considerate in relazione al criterio della normalità media e rilevate attraverso dati statistici ed elementi scientifici e di comune esperienza. Ne consegue che la causalità generale tra un'attività e un evento dannoso è solo idonea a far ritenere tale attività pericolosa, ma non anche a far ritenere che il danneggiato abbia assolto al suo obbligo di provare il nesso causale tra tale attività e l'evento, in quanto, ove si ritenesse ciò, la sola esistenza dell'attività pericolosa comporterebbe l'esisten- za del nesso causale, gravando poi sul soggetto esercente l'attività provare che nel caso concreto o non esiste tra detta attività e lo specifico evento alcun nesso causale ovvero fornire la prova liberatoria della sua responsabilità, nei termini di cui all'art. 2050 c.c. (aver adottato tutte le misure idonee ad evitare il danno)» [20].

Dato per consolidato il principio che vede l'applicazione ai casi di risarcimento danni da farmaci difettosi, dell'art. 2050 c.c., occorre sottolineare alcuni aspetti precipui della disciplina codicistica. Innanzitutto, si tratta di un'ipotesi di presunzione iuris tantum e non di responsabilità oggettiva come le disposizioni immediatamente vicine, ovvero gli artt. 2049 e 2051 c.c. Infatti si legge nella giurisprudenza che «in tema di responsabilità per esercizio di attività pericolosa l'art. 2050 c.c. pone una la presunzione di colpa a carico del danneggiante» [21]. Tale presunzione di responsabilità è posta, comunque a carico di chi materialmente svolge l'attività pericolosa e non di colui per conto del quale l'attività viene svolta.

Ciò che rileva è che l'attività presenti una notevole potenzialità di danno a terzi mentre nulla rileva se l'attività, normalmente innocua, diventa pericolosa per la condotta di chi la esercita; infatti, non rileva «una mera condotta soggettiva pericolosa, idonea a far sorgere la responsabilità soltanto secondo la regola dell'art. 2043 c.c.» [22-24].

Tutto questo ha un'enorme rilevanza processuale perché nel caso dell'art. 2050 c.c. diventa necessario accertare di fatto la natura dell'attività svolta 0 il grado di efficienza dei mezzi utilizzati [22].

Ma occorre chiarire che la presunzione si applica sia alle attività oggettivamente pericolose sia a quelle che pur non essendolo diventano pericolose in conseguenza del tipo di strumenti adottati dall'esercente. E così la suprema corte ha chiarito che «la pericolosità ex art.2050 c.c. sussiste non solo nel caso in cui la probabilità dell'accadimento dannoso deriva da un'azione, ma anche quando deriva dall'omissione di cautele che in concreto sarebbe stato necessario adottare in relazione alla natura dell'attività esercitata alla stregua delle norme di comune diligenza e prudenza» [25-26]. In effetti, vengono così introdotti due criteri per la valutazione della natura pericolosa: la natura intrinseca dell'attività e la qualità dei mezzi adoperati. Peraltro la Suprema Corte ha specificato che la pericolosità di un'attività va apprezzata, per gli effetti di cui all'art. 2050 c.c., esclusivamente in relazione alla probabilità delle conseguenze dannose che possano derivarne e non anche in riferimento alla diffusione delle modalità con le quali viene comunemente esercitata, che ben potrebbero essere tutte e sempre inadeguate, senza per questo elidere i presupposti per l'applicazione della norma citata [27]. Naturalmente il giudizio di pericolosità non va espresso sulla base dell'evento dannoso effettivamente verificatosi, ma secondo una "prognosi postuma" che il giudice deve compiere sia sulla 
base di nozioni desunte dalla comune esperienza, sia tenuto conto delle circostanze di fatto che si presentavano al momento dell'esercizio dell'attività ed erano conoscibili dall'uomo medio, o comunque dovevano essere conosciute dall'agente in considerazione del tipo di attività esercitata [28]. Alla luce di quanto sinora detto seppur in breve, la posizione processuale di una società farmaceutica è delicata, complessa e va analizzata attentamente perché nulla può essere dato per scontato né da una parte né dall'altra specie alla luce di una giurisprudenza sempre in continua evoluzione.

\section{DISCLOSURE}

Gli Autori dichiarano di non avere conflitti di interesse di natura finanziaria.

\section{BIBLIOGRAFIA}

1. Perucca E, Albani F, Capovilla G, Bernardina BD, Michelucci R, Zaccara G. Recommendations of the Italian League against Epilepsy working group on generic products of antiepileptic drugs. Epilepsia 2006; 47: 16-20

2. Kesselheim AS, Misono AS, Lee JL, Stedman MR, Brookhart MA, Choudhry NK et al. Clinical equivalence of generic and brandname drugs used in cardiovascular disease: a systematic review and meta-analysis. JAMA 2008; 300: 2514-26

3. Figueiras MJ, Marcelino D, Cortes MA. People's views on the level of agreement of generic medicines for different illnesses. Pharm World Sci 2008; 30: 590-4

4. TAR Lazio Roma, sez II-bis, 18 dicembre 2008, n. 11697

5. R.D. 27 luglio 1934 , n. 1265

6. Cassazione civile, sez. II, sentenza n. 8073 del 28 marzo 2008

7. App. Roma, sez. I, 8 febbraio 2010

8. Cassazione civile, sezioni unite, sentenza n. 576 del 11 gennaio 2008

9. Cassazione civile, sentenza n. 574-586 del 11 gennaio 2008

10. Cassazione civile, sentenza n. 8069 del 20 luglio 1993

11. Cassazione civile, sentenza n. 1138 del 1 febbraio 1995

12. D.Lgs. n. 206 del 6 settembre 2005

13. Tribunale di Messina, 17 marzo 2005

14. Tribunale di Roma, 20 aprile 2002

15. Cassazione civile, sez. III, sentenza n. 814 del 27 gennaio 1997

16. Cassazione civile, sez. III, sentenza n. 8395 del 27 luglio 1991

17. Cassazione civile, sez. III, sentenza n. 6241 del 15 luglio 1987

18. App. Roma, sez. III, sentenza del 2 dicembre 2008

19. Cassazione civile, sentenza n. 11275 del 27 maggio 2005

20. Cassazione civile, sezioni unite, sentenza n. 582 del 11 gennaio 2008

21. Cassazione civile, sentenza n. 10382 del 17 luglio 2002

22. Cassazione civile, sentenza n. 20357 del 21 ottobre 2005

23. Cassazione civile, sentenza n. 15334 del 15 ottobre 2004

24. Cassazione civile, sentenza n. 13530 del 21 dicembre 1992

25. Cassazione civile, sentenza n. 10300 del 7 maggio 2007

26. Cassazione civile, sentenza n. 5341 del 29 maggio 1998

27. Cassazione civile, sez. III, sentenza n. 7916 del 26 aprile 2004

28. Cassazione civile, sez. III, sentenza n. 9205 del 30 agosto 1995

\section{CORRESPONDING AUTHOR}

Per la parte clinica: Dott.ssa Luciana Pazzagli; e-mail: luciana.pazzagli@asf.toscana.it Per la parte legale: Avv. Marianna Pulice; e-mail: avv.pulice@alice.it 